\title{
INVESTIGACIONES
}

\section{Contexto socioeconómico, práctica pedagógica y aprendizaje autónomo en el aula*}

\author{
Socioeconomic context, pedagogical practice \\ and autonomous learning in the classroom
}

\author{
Marco Antonio Villalta Paucar ${ }^{a}$, Cecilia Assael Budnik ${ }^{b}$ \\ ${ }^{a}$ Escuela de Psicología, Universidad de Santiago de Chile. \\ Correo electrónico: marco.villalta@usach.cl \\ ${ }^{\text {b}}$ Facultad Educación, Universidad Autónoma de Chile. \\ Correo electrónico: cecilia.assael@uautonoma.cl.
}

\begin{abstract}
El objetivo fue analizar la práctica pedagógica efectiva para el logro de aprendizaje autónomo en diferentes contextos socioeducativos de Educación Básica. Metodológicamente es un estudio descriptivo de las clases de ocho profesoras, 16 directivos y 40 niños de primero y segundo básico, de cuatro escuelas de la Región Metropolitana de Santiago de Chile, seleccionadas según nivel socioeconómico (NSE) y logro escolar medido por la prueba del Sistema de Medición de la Calidad de la Educación (SIMCE). Se hizo registro fílmico de las clases y entrevistas. Se realizó análisis de la Conversación (AC), de criterios de Experiencia de Aprendizaje Mediado (EAM), estadística no paramétrica, y análisis de contenido semántico. Los resultados indican que las prácticas pedagógicas de aula son diferentes según el NSE y el logro escolar. La eficacia para promover aprendizajes autónomos se orienta a la nivelación o de enriquecimiento cultural según sea el nivel socioeconómico de los alumnos.
\end{abstract}

Palabras clave: logro escolar, intercambio, segregación académica, mediación, análisis de la conversación, sala de clase.

\section{ABSTRACT}

The aim of this research was to analyze the effective pedagogical practice for the achievement of autonomous learning in different socio-educational contexts of Elementary Education. Methodology consisted of a descriptive study of the classes of eight teachers, 16 executives and 40 children of first and second grade from four schools of the Metropolitan Region, Chile, selected according to their socioeconomic status (SES) and academic achievement measured by the test of the Education Quality Measurement System (SIMCE). Classes and interviews were recorded. Conversation Analysis (CA), criteria for the Mediated Learning Experience (MLE), nonparametric statistics, and semantic content were analyzed. Findings suggest that the classroom pedagogical practices are different according to the SES and academic achievement. The effectiveness to promote autonomous learning is focused on the placement or cultural enrichment according to students' socioeconomic status.

Keywords: academic achievement, exchange, academic segregation, mediation, conversation analysis, classroom.

Patrocinado por el Fondo Nacional de Desarrollo Científico y Tecnológico (FONDECYT) del gobierno de Chile. Proyecto FONDECYT 1150237 


\section{ANTECEDENTES}

Diversos estudios reiteran en señalar que aspectos como el contexto socioeconómico y cultural en que se encuentra inserta la escuela, inciden en los discursos, prácticas pedagógicas y resultados escolares (Bruns et al. 2014; Hennessy et al. 2016; Sánchez y Amigo 2016; Villalta y Palacios 2014).

A pesar de las evidentes diferencias estructurales y socioculturales de las escuelas (De Gregorio y Bruns 2016; Laboratorio Latinoamericano de Evaluación de la calidad de la Educación 2010), las metas de logro de aprendizaje son similares para todos, en Chile medido por la prueba del Sistema de Medición de la Calidad de la Educación (SIMCE) que asegura los estándares de aprendizaje nacionales y comparables internacionalmente (Agencia de Calidad de la Educación 2015); en relación a los cuales las prácticas pedagógicas de aula se articulan de cara a sus realidades específicas y con resultados contingentes (Martinic y Villalta 2016; Volante, Cumsille, Denardin y Müller 2008).

Las condiciones socioeconómicas y sus correlatos psicosociales son elementos del contexto que inciden en lo que hace y logra la escuela. En efecto, los estudios en Latinoamérica reiteran en señalar que las inequidades socioeconómicas, la vulnerabilidad social de los alumnos, las condiciones sociodemográficas de las escuelas, las atribuciones y expectativas educativas entre otras, se reflejan en los logros de aprendizaje (Cornejo y Redondo 2007; Donoso y Arias 2012; Murillo y Román 2009). No obstante, no se puede establecer una relación causal entre las condiciones del contexto y lo que pueden hacer y logran las escuelas y la comunidad educativa; los estudios han evidenciado experiencias educativas donde la situación de pobreza y/o vulnerabilidad social no ha sido obstáculo para alcanzar logros de aprendizaje escolar cuando se han implementado adecuadas acciones de mejoramiento (UNICEF 2004; Volante et al. 2008).

De las diversas aproximaciones a definir práctica pedagógica y escuelas efectivas (Cornejo y Redondo 2007; Godoy, Varas, Martínez, Treviño y Meyer 2016), vale destacar que reconocen el carácter contextual de la práctica y cuyos logros tienen que ver con elementos como involucrar a los estudiantes en las actividades de la clase, la gestión del orden favorable a la transmisión de contenidos y la generación de buen clima social de aula, entre otros (De Gregorio y Bruns 2016; Godoy et al. 2016; Treviño, Varas, Godoy y Martinez 2016). Estos elementos confluyen en estructuras de interacción dialogal en la sala de clase contextualizadas.

Desde la perspectiva socio-histórico-cultural vygotskiano, el educador tiene la potencialidad de ser mediador de la semiótica cultural para la formación de los procesos psicológicos superiores de sus educandos (Damianova y Sullivan 2011; Shreyar, Zolkower y Pérez 2010). En dicha línea, la interacción dialogal educativa es una mediación entre el educando y el entorno que promueve el educador para favorecer en los educandos la autonomía de aproximarse de modo directo los saberes de la cultura (Feuerstein, Rand, Hoffman, Egozi y Shachar-Segev 1991; Poehner y Infante 2015; Tébar 2007; Tzuriel y Shamir 2007).

El aprendizaje autónomo refiere a que las personas piensen por sí mismas y sean capaces de asumir la responsabilidad de su proceso de apropiarse de la cultura, de transformarla y transformarse en dicho proceso. En tal sentido, la autonomía implica independencia respecto a otros y el desarrollo de la autorregulación en tanto dimensión procedimental de los procesos metacognitivos (Moreno y Martínez 2007; Osses y Jaramillo 2008). En la perspectiva de la teoría de modificabilidad estructural cognitiva de Reuven Feuerstein, la aproximación al 
aprendizaje autónomo tiene relación con la capacidad del individuo de beneficiarse de modo directo de la exposición a situaciones de aprendizaje (Feuerstein 1990).

El contexto sociocultural no predefine el tipo de dialogo en el aula, no obstante, entrega los principios simbólicos que enmarcan las posibilidades de la interacción dialogal (Vidal 2009; Villalta 2017). Así, en una perspectiva sociocultural del aprendizaje y el desarrollo humano, la práctica pedagógica eficaz no solamente gestiona y transmite contenidos de aprendizaje indicados en el currículum; también de modo tácito e imbricado a dicha actividad, modifica la estructura -dialogal- de aprendizaje, la propensión para aprender (Feuerstein 1990; Villalta 2017).

En el presente estudio se relacionan dos aproximaciones teóricas de estudio que comparten una aproximación socio histórico y cultural a los procesos cognitivos: el análisis de la conversación (AC) en la sala de clase (Villalta 2009; Villalta y Martinic 2013) y la teoría de Experiencias de Aprendizaje Mediado (EAM) de Reuven Feuerstein (Feuerstein 2006; Feuerstein et al. 1991)

Las estructuras de conversación se organizan a lo largo de la clase para desarrollar el currículum y junto a ello promover el desarrollo cognitivo de los estudiantes (Martinic y Villalta 2016; Villalta y Martinic 2013). La intervención con que educador inicia un diálogo define el tipo de demanda cognitiva que realiza a sus alumnos (Cuadrado y Fernández 2008; Tornero, Ramaciotti, Truffello, y Valenzuela 2015; Wells y Mejia 2005). Dicha intervención se inserta en la estructura de conversación básica en la clase InicioRespuesta-Cierre/Evaluación y la investigación al respecto reconoce variantes según el contexto, la interactividad, el objetivo pedagógico, entre otros aspectos (Emanuelsson y Sahlström 2008; Lehesvuori 2013; Nathan y Kim 2009).

Por su parte la perspectiva de la teoría de Experiencia de Aprendizaje Mediado (EAM) desarrollada por Feuerstein (Feuerstein 2006; Kozulin 1999; Tzuriel 2010) permite reconocer situaciones e intervenciones del profesor que destacan su rol como mediador afectivo y efectivo del saber (Poehner y Infante 2015; Tocaimaza-Hatch 2016). Y dichos criterios se ponen de manifiesto en la interacción verbal (Assael 2015; Villalta, Martinic y Assael 2013). La teoría EAM reconoce criterios de mediación Universales, propio toda relación educativa que promueve el aprendizaje, y Diferenciadores, que son específicos para cada circunstancia del aprendiz, para mejorar la calidad de la interacción del educando con el saber cultural y la modificabilidad estructural del aprendizaje (Feuerstein 1990; Orrú 2003; Tzuriel 2010).

Entonces, hay elementos del contexto sociocultural -la cultura escolar, el nivel socioeconómico, son algunos de ellos- que inciden en lo que sucede y se dice sobre lo que sucede en el aula, ¿las estructuras de interacción mediadas para promover el aprendizaje autónomo en las aulas, serán diferentes según el logro escolar y nivel socioeconómico de la Escuela?; ¿la historia de logro o fracaso escolar de la Escuela incidirá en los discursos y las prácticas de aula? El presente estudio tiene por objetivo analizar la práctica pedagógica efectiva para el logro de aprendizaje autónomo en diferentes contextos socioeducativos de Educación Básica.

\section{METODOLOGÍA}

Se realiza una investigación descriptiva, en un paradigma interpretativo de discursos y acciones de los actores educativos que combina técnicas de recolección y análisis de datos 
cuantitativos de tipo no paramétrico, y cualitativas de análisis de registros etnográficos de observación de clases, y análisis de contenido del discurso recogido el año 2015.

Es un estudio de casos con ocho profesoras, 16 directivos y 40 niños correspondientes a cuatro escuelas seleccionadas según: (1) NSE Alto, medio, y Bajo; (2) Logro SIMCE 2014 en comprensión lectora Alto/Bajo en 2do Básico comparados con el promedio nacional; (3) Experiencia docente en la escuela: Novata (menos de 3 años) / Experta (más de 3 años) y (4) historia de segregación intra-académica, esto es, agrupamiento de los alumnos al interior de la escuela según su desempeño escolar, para esto se toma como referencia el estudio auspiciado por Fondo de Investigación y Desarrollo en Educación (FONIDE) de Chile, realizado por Ernesto Treviño con la base de datos nacional de escuelas chilenas (Treviño, Valenzuela y Villalobos 2014b). Se realizaron entrevistas con profesoras, grupos focales con directivos y alumnos de 1 ro y 2 do básico.

Con estos criterios se seleccionaron cuatro escuelas (Tabla 1): Escuela SFF de nivel socioeconómico Medio y SIMCE Alto (SSF / NSE Medio / SIMCE Alto): (2) Escuela VL de Nivel socioeconómico Bajo y SIMCE Bajo (VL / NSE Bajo / SIMCE Bajo): (3) Escuela FL, de nivel socio cómico Medio-Alto y SIMCE Alto (FL / NSE Medio Alto / SIMCE Alto); y, (4) Escuela SPQ de nivel socioeconómico Medio y SIMCE Bajo (SPQ / NSE Medio / SIMCE Bajo). Cabe señalar que las escuelas con historia de segregación intra-académica son las escuelas de nivel socioeconómico Medio uno de SIMCE Alto y el otro de logro SIMCE Bajo.

Tabla 1. Descripción de los datos según Nivel Socioeconómico (NSE), Actor Educativo, categorización SIMCE

\begin{tabular}{|c|c|c|c|c|c|c|}
\hline \multicolumn{7}{|c|}{ SIMCE } \\
\hline NSE & Actor & & Alto & Bajo & Total & Técnica \\
\hline \multirow{4}{*}{ Medio Alto } & \multirow{2}{*}{ Profesora } & Experta & 1 & & 1 & Registro de clase y entrevista \\
\hline & & Novato & 1 & & 1 & Registro de clase y entrevista \\
\hline & Directivo & & 2 & & 2 & Grupo focal \\
\hline & Niños & & 10 & & 10 & Grupo focal \\
\hline \multirow{4}{*}{ Medio } & \multirow{2}{*}{ Profesora } & Experta & 1 & 1 & 2 & Registro de clase y entrevista \\
\hline & & Novato & 1 & 1 & 2 & Registro de clase y entrevista \\
\hline & Directivo & & 2 & 2 & 4 & Grupo focal \\
\hline & Niños & & 10 & 10 & 20 & Grupo focal \\
\hline \multirow{4}{*}{ Bajo } & \multirow{2}{*}{ Profesora } & Experta & 1 & & 1 & Registro de clase y entrevista \\
\hline & & Novato & 1 & & 1 & Registro de clase y entrevista \\
\hline & Directivo & & 2 & & 2 & Grupo focal \\
\hline & Niños & & 10 & & 10 & Grupo focal \\
\hline TOTAL & & & 42 & 14 & 56 & \\
\hline
\end{tabular}


El procedimiento del estudio consideró criterios éticos de la investigación social de información a los actores educativos, respeto a la voluntad de participación y confidencialidad; esto concreto en cartas de Autorización de los equipos directivos, Cartas de Consentimiento Informado de las profesoras participantes y de Asentimiento informado de los apoderados de los alumnos y alumnas de 1ro y 2do Básico. Dichas cartas fueron validadas por el comité de ética de la unidad patrocinante del estudio.

Para el análisis de los registros de observación de aula se consideró estudios previos sobre las estructuras dialogales o intercambios verbales entre profesor y alumnos donde se tipifican seis tipos de intercambios en la clase (Villalta-Paucar, Martinic-Valencia, AssaelBudnik y Aldunate-Ruff 2018; Villalta 2014):

(1) Expositivo, estructura dialogal unidireccional de la profesora al alumno

(2) Regulativo, estructura dialogal que inicia la profesora para restablecer los turnos de habla en la clase;

(3) Co-formado, estructura dialogal que inicia la profesora para evaluar contenidos previamente presentados;

(4) Explicativo, estructura dialogal que inicia el alumno para solicitar información o retroalimentación a la profesora sobre sus actividades escolares;

(5) Cooperativo, estructura dialogal donde profesora y alumnos participan activamente en la co-construcción del contenido y,

(6) Colaborativo, estructura dialogal donde profesora y alumnos intervienen definiendo el objetivo y condiciones de satisfacción de la conversación respecto a los contenidos de la clase.

Los registros de diálogo en el aula también fueron analizados con los criterios de Experiencia de Aprendizaje Mediado (EAM). Para el presente estudio se consideraron los tres criterios Universales ya descritos en otros estudios en relación a los intercambios verbales (Assael-Budnik y Aldunate-Ruff 2018; Villalta 2014; Villalta et al. 2013). Estos son:

(1) Intencionalidad y Reciprocidad, donde la profesora explicita a los alumnos el objetivo de su acción y logra que ellos participen en la interacción propuesta.

(2) Significado, donde, en términos operativos, la profesora pone de manifiesto sus propias razones, preguntas y motivaciones del por qué y para qué de la actividad de enseñanza.

(3) Trascendencia, donde la profesora promueve que la interacción vivenciada en el aula sea transferida por los alumnos a situaciones más allá del aquí y del ahora de la clase.

Las categorías de observación fueron codificadas por observadores expertos entrenados en las mismas con uso del software Videograph (Rimmele 2009). El análisis de concordancia inter-observador medido por la prueba Kappa que va de $0=$ nula concordancia a $1=$ Total concordancia; indica que la concordancia inter-observador fue entre .89 y .99 para los criterios EAM, y entre .92 y .93 en las categorías de Intercambio, lo que es valorado como Considerable y Casi Perfecta (Landis y Koch 1977).

El análisis de los registros de observación fue abordado con estadística no paramétrica para analizar la asociación entre categorías con las pruebas Chi cuadrado de Pearson, y, 
en el caso de encontrar asociación significativa entre las categorías analizadas, se utilizó el coeficiente $\mathrm{V}$ de Cramer para determinar la fuerza de dicha relación, que puede ir de 0 = débil relación a $1=$ fuerte relación; en el presente estudio se considera relación de fuerza grande el valor sobre .65; adecuada o moderada el valor por sobre .30; débil bajo .29 y nula bajo .10. En caso de asociación significativa y de fuerza moderada, se analizaron las casillas donde se ubica con mayor fuerza la asociación, esto a través del cálculo de los Residuos corregidos ( $\mathrm{z} \pm 1.96)$.

El análisis de los discursos se realizó con principios del análisis de contenidos semántico (Navarro y Díaz 1999; Piñuel 2002), determinando unidades de registro definidos por palabras-termino que se fueron refinando en un proceso inductivo de categorización de los registros discursivos orientado por el objetivo del estudio. El análisis de contenido se realizó con el software NVivo 11 Pro.

\section{RESULTADOS}

\subsection{OBSERVACIÓN DE LAS PRÁCTICAS PEDAGÓGICAS EN EL AULA}

Tabla 2. Comparación de marcas de tiempo de Intercambios, EAM Universal por profesoras con prueba de Kruskal Wallis

\begin{tabular}{|c|c|c|c|c|}
\hline \multirow[b]{2}{*}{ Profesora } & \multicolumn{2}{|c|}{ Intercambio } & \multicolumn{2}{|c|}{ EAM Universal } \\
\hline & $\mathrm{N}$ & Rango promedio & $\mathrm{N}$ & Rango promedio \\
\hline RSSF & 1751 & 4022,21 & 64 & 538,54 \\
\hline MISSF & 1195 & 4750,41 & & \\
\hline EVL & 1324 & 3813,41 & 102 & 459,18 \\
\hline SVL & 709 & 5300,97 & 64 & 214,00 \\
\hline $\mathrm{CF}$ & 1145 & 5073,23 & 92 & 622,90 \\
\hline $\mathrm{MF}$ & 1000 & 3763,34 & 287 & 651,40 \\
\hline ASPQ & 943 & 3618,94 & 245 & 238,53 \\
\hline TSPQ & 702 & 5612,90 & 60 & 340,93 \\
\hline Total & 8769 & & 914 & \\
\hline Chi-cuadrado & \multicolumn{2}{|r|}{654,450} & \multicolumn{2}{|r|}{507,286} \\
\hline gl & \multicolumn{2}{|r|}{7} & \multicolumn{2}{|r|}{6} \\
\hline Sig. asintótica & \multicolumn{2}{|r|}{.001} & \multicolumn{2}{|r|}{.001} \\
\hline
\end{tabular}


El análisis por profesor (Tabla 2) indica que los intercambios, EAM Universales son diferentes entre las ocho profesoras. Es decir, cada diálogo y tipo de mediación responde a sus propias circunstancias y contingencias.

Tabla 3. Comparación de Intercambios,

EAM Universal por Experiencia docente con la prueba U de Mann-Whitney

\begin{tabular}{|c|c|c|c|c|c|c|c|}
\hline \multicolumn{2}{|c|}{$\begin{array}{l}\text { Experiencia } \\
\text { docente }\end{array}$} & $\mathrm{N}$ & $\begin{array}{l}\text { Rango } \\
\text { promedio }\end{array}$ & $\begin{array}{c}\text { Suma } \\
\text { de rangos }\end{array}$ & $\begin{array}{c}\text { U de } \\
\text { Mann-Whitney }\end{array}$ & $\mathrm{Z}$ & $\begin{array}{l}\text { Sig. asintótica } \\
\text { (bilateral) }\end{array}$ \\
\hline \multirow{3}{*}{ Intercambios } & Experta & 2904 & 3701,85 & 10750165,50 & \multirow{3}{*}{5722774,5} & \multirow{3}{*}{$-4,884$} & \multirow{3}{*}{0,000} \\
\hline & Novata & 4220 & 3466,61 & 14629084,50 & & & \\
\hline & Total & 7124 & & & & & \\
\hline \multirow{3}{*}{ EAM Universal } & Experta & 351 & 311,85 & 109458,50 & \multirow{3}{*}{42875,5} & \multirow{3}{*}{$-1,216$} & \multirow{3}{*}{0,224} \\
\hline & Novata & 258 & 295,68 & 76286,50 & & & \\
\hline & Total & 609 & & & & & \\
\hline
\end{tabular}

Tabla 4. Comparación de marcas de tiempo de Intercambios, EAM Universal por Nivel Socioeconómico de Escuela con prueba de Kruskal Wallis

\begin{tabular}{|c|c|c|c|c|c|c|}
\hline Categorías & $\begin{array}{c}\text { Nivel } \\
\text { socioeconómico }\end{array}$ & $\mathrm{N}$ & $\begin{array}{l}\text { Rango } \\
\text { promedio }\end{array}$ & Chi-cuadrado & $\mathrm{gl}$ & $\begin{array}{c}\text { Sig. } \\
\text { asintótica }\end{array}$ \\
\hline \multirow{4}{*}{ Intercambios } & Medio Alto & 2145 & 3632,75 & \multirow{3}{*}{3,817} & \multirow{3}{*}{2} & \multirow{3}{*}{0,148} \\
\hline & Medio & 2946 & 3528,79 & & & \\
\hline & Bajo & 2033 & 3537,23 & & & \\
\hline & Total & 7124 & & & & \\
\hline \multirow{7}{*}{ EAM Universal } & Medio Alto & 379 & 360,98 & \multirow{3}{*}{142,411} & \multirow{3}{*}{2} & \multirow{3}{*}{0,000} \\
\hline & Medio & 64 & 294,77 & & & \\
\hline & Bajo & 166 & 181,13 & & & \\
\hline & Total & 609 & & & & \\
\hline & Medio & 2028 & 2198,07 & & & \\
\hline & Bajo & 1762 & 2584,60 & & & \\
\hline & Total & 5104 & & & & \\
\hline
\end{tabular}

No obstante, al agrupar datos por Experiencia docente y Nivel Socioeconómico (Tablas 2 y 3 ) estos ponen en evidencia que la Experiencia docente es diferente en el tipo de diálogos 
posibles en la clase y no el nivel socioeconómico; y, a la inversa, la experiencia de docencia no incide en el tiempo que se usan criterios de mediación Universales y Diferenciadores en el aula, pero estos son diferentes al compararlos por nivel socioeconómico.

Tabla 5. Asociación entre EAM Universales y Diferenciadores con Escuelas según Nivel socioeconómico y logro SIMCE

\begin{tabular}{|c|c|c|c|c|c|c|c|}
\hline & & & \multicolumn{4}{|c|}{ Escuela / NSE / SIMCE } & \multirow[b]{2}{*}{ Total } \\
\hline & & $\begin{array}{l}\text { Datos observados } \\
\text { y corregidos }\end{array}$ & $\begin{array}{c}\text { SSF / NSE } \\
\text { Medio / } \\
\text { SIMCE } \\
\text { Alto }\end{array}$ & $\begin{array}{c}\text { VL / NSE } \\
\text { Bajo / } \\
\text { SIMCE } \\
\text { Bajo }\end{array}$ & $\begin{array}{c}\text { FL / NSE } \\
\text { Medio Alto } \\
\text { / SIMCE } \\
\text { Alto }\end{array}$ & $\begin{array}{c}\text { SPQ / NSE } \\
\text { Medio / } \\
\text { SIMCE } \\
\text { Bajo }\end{array}$ & \\
\hline \multirow{7}{*}{$\begin{array}{c}\text { EAM } \\
\text { Universales }\end{array}$} & \multirow[b]{2}{*}{ Intencionalidad } & Recuento & 20 & 116 & 20 & 271 & 427 \\
\hline & & $\begin{array}{l}\text { Residuos } \\
\text { corregidos }\end{array}$ & $-2,6$ & 6,6 & $-21,1$ & 18,1 & \\
\hline & \multirow{2}{*}{ Significado } & Recuento & 13 & 9 & 132 & 20 & 174 \\
\hline & & $\begin{array}{l}\text { Residuos } \\
\text { corregidos }\end{array}$ & 0,3 & $-4,9$ & 10,2 & $-6,8$ & \\
\hline & \multirow{2}{*}{ Trascendencia } & Recuento & 31 & 41 & 227 & 14 & 313 \\
\hline & & $\begin{array}{l}\text { Residuos } \\
\text { corregidos }\end{array}$ & 2,5 & $-2,9$ & 13,8 & $-13,4$ & \\
\hline & Total & Recuento & 64 & 166 & 379 & 305 & 914 \\
\hline
\end{tabular}

Se encuentra asociación entre los EAM Universales con la Escuela [Chi Cuadrado $(6, \mathrm{~N}=914)=527,7 ; \mathrm{p}<.001]$, con fuerza de asociación Moderada (V de Cramer $=.54)$. El análisis de residuos tipificados indica que el criterio de EAM Universal de Intencionalidad es más alto en las Escuelas que comparten el tipo de SIMCE Bajo; y el criterio EAM Trascendencia en las escuelas que comparten el logro de SIMCE Alto. Aunque se encuentra asociación significativa entre los Intercambios con las Escuelas [Chi Cuadrado $(15, \mathrm{~N}=8769)=845,85 ; \mathrm{p}<.001]$ la fuerza de la asociación es débil $(\mathrm{V}$ de Cramer $=.18)$. 
Tabla 6. Asociación entre EAM Universales y tipos de Intercambio en cuatro escuelas

\begin{tabular}{|c|c|c|c|c|c|}
\hline \multirow[b]{2}{*}{ Intercambios } & \multirow{2}{*}{$\begin{array}{c}\text { Datos observados y } \\
\text { corregidos }\end{array}$} & \multicolumn{3}{|c|}{ EAM Universales } & \multirow{2}{*}{ Total } \\
\hline & & Intencionalidad & Significado & Trascendencia & \\
\hline \multirow{2}{*}{ Expositivo } & Recuento & 425 & 135 & 172 & 732 \\
\hline & Residuos corregidos & 12,1 & $-0,3$ & $-12,1$ & \\
\hline \multirow{2}{*}{ Co-formado } & Recuento & 56 & 29 & 67 & 152 \\
\hline & Residuos corregidos & $-1,6$ & 0,1 & 1,5 & \\
\hline \multirow{2}{*}{ Regulativo } & Recuento & 74 & 5 & 17 & 96 \\
\hline & Residuos corregidos & 7,0 & $-3,5$ & $-4,3$ & \\
\hline \multirow{2}{*}{ Explicativo } & Recuento & 29 & 0 & 9 & 38 \\
\hline & Residuos corregidos & 4,2 & $-3,0$ & $-1,9$ & \\
\hline \multirow{2}{*}{ Cooperativo } & Recuento & 10 & 50 & 131 & 191 \\
\hline & Residuos corregidos & $-11,3$ & 2,8 & 9,2 & \\
\hline \multirow{2}{*}{ Colaborativo } & Recuento & 0 & 41 & 138 & 179 \\
\hline & Residuos corregidos & $-12,4$ & 1,5 & 11,4 & \\
\hline Total & Recuento & 594 & 260 & 534 & 1.388 \\
\hline
\end{tabular}

Dado que los EAM Diferenciadores por su naturaleza son propios de cada contexto, interesa conocer la relación entre los EAM Universales con los intercambio (Tabla 6). Se encuentra asociación significativa [Chi Cuadrado (10, N=1388)=845,848; $\mathrm{p}<.001$ ] y fuerza de asociación Moderada (V de Cramer = .39) entre los tipos de Intercambio y los EAM Universales. El análisis de los residuos tipificados corregidos indica que la fuerza mayor de la asociación es entre Intercambio Expositivo y EAM Universal Intencionalidad, y entre Intercambio Colaborativo y EAM Universal Trascendencia.

En síntesis, las prácticas pedagógicas en el aula responden privilegiadamente a sus respectivos contextos inmediatos, la experiencia docente incide en el tipo de diálogos posibles, pero no es diferente al verlo por nivel socioeconómico; los EAM Universales y Diferenciadores no son diferentes según la experiencia docente, pero sí lo son respecto al nivel socioeconómico de las escuelas. Así, la Escuela de Alto logro SIMCE y medio-alto nivel socioeconómico están asociadas a mayor empleo de EAM Universal Trascendencia, el cual está asociado a los intercambios de tipo Colaborativo, y la escuela de Bajo logro SIMCE y bajo nivel socioeconómico está asociada a mayor empleo de EAM Intencionalidad, el cual se encuentra mayormente asociado a los intercambios de tipo Expositivos. 


\subsection{LOS DISCURSOS SOBRE LA PRÁCTICA}

Dado que, sustancialmente, cada actor responde a su contexto, interesa conocer desde los discursos qué ponen de relieve sobre las prácticas pedagógicas que favorezcan la autonomía para aprender. Para esto se categoriza las opiniones de directivos, profesores y alumnos de primero y segundo básico en dos núcleos temáticos: (1) Metas de aprendizaje y (2) Diversidad de los alumnos.

\subsubsection{Las Metas de aprendizaje}

Las Metas de aprendizaje refieren a juicios que los actores reportan como elemento que les permite afirmar que se han logrado cambios deseados por la acción de enseñar y aprender (referidos a metodologías, planificación, pertinencia cultural, entre otros). Se organizaron los discursos en cuatro Metas de Aprendizaje:

Alcanzar logros escolares: Referido a logros de dominio de contenidos. Por ejemplo, referido a indicadores de aprendizaje de lecto-escritura y operaciones básicas:

“...ellas van aprendiendo...porque yo les muestro su cuaderno que tenían al principio, cómo escribían antes y cómo escriben ahora..." (Profesora Novata, Escuela FL, NSE Medio-Alto, SIMCE Alto).

“...la profesora me saco y yo estoy explicando que $8+8$ son 16 y que $1+1$ son 2 " (Alumno Iro Básico, Escuela SSF, NSE Medio, SIMCE Alto).

Ser buenas personas: Afirmaciones generales sobre el sentido de la educación. Por ejemplo:

“... ¿para qué sirve la escuela? Para formar buenas personas, si eso es en definitiva; que uno sepa más y que otro sepa menos la verdad es que cualitativamente puede ser importante, pero cuantitativamente...perdón, cuantitativamente puede ser importante, pero cualitativamente no lo es para nada..." (Profesora Experto, Escuela SPQ NSE Medio, SIMCE Bajo).

Objetivos Transversales: Que pone en evidencia los valores de convivencia escolar señalados en el marco curricular. Por ejemplo:

“...Respeto, valores...valores...yo les hablo mucho de respeto, de la empatía, de ponerse en el lugar de otro, trato de ponerlos en situaciones "mira si tú haces tal cosa mira lo que provocas” (Profesora Experta, Escuela VL, NSE Bajo, SIMCE Bajo).

“...Nos dice cosas sobre los inmigrantes, y a ser respetuosos porque hay que ser respetuosos y tener limpia la sala para poder trabajar mejor...” (Alumna 2do Básico, Escuela SPQ, NSE Medio, SIMCE Bajo).

Disposición para aprender: que refiere a promoción de habilidades o competencias donde se observa que estudiante aplica los contenidos pedagógicos a situaciones cotidianas, 
o manifiesta agrado por aprender. Por ejemplo:

“...(En) matemáticas el ir al kiosko y comprar, eso sí les sirve mucho, porque sin darse cuenta ellos están sumando y restando, cuando reciben vuelto, cuando pagan, así que eso pienso yo que les ayudaría mucho" (Profesora Experta, Escuela SSF de NSE Medio, SIMCE Alto).

“...Que me gustan los números solo que no aprendo matemáticas; pero ese día estaba rápida haciendo eso y portándome bien” (Alumna 2do Básico, Escuela VL, NSE Bajo, SIMCE Bajo).

Se codifican y organizan las referencias en las categorías mencionadas; según la cantidad de las mismas se grafica la tendencia de los discursos según la historia de segregación intra-académica (Figura 1) y NSE de la Escuela (Figura 2).

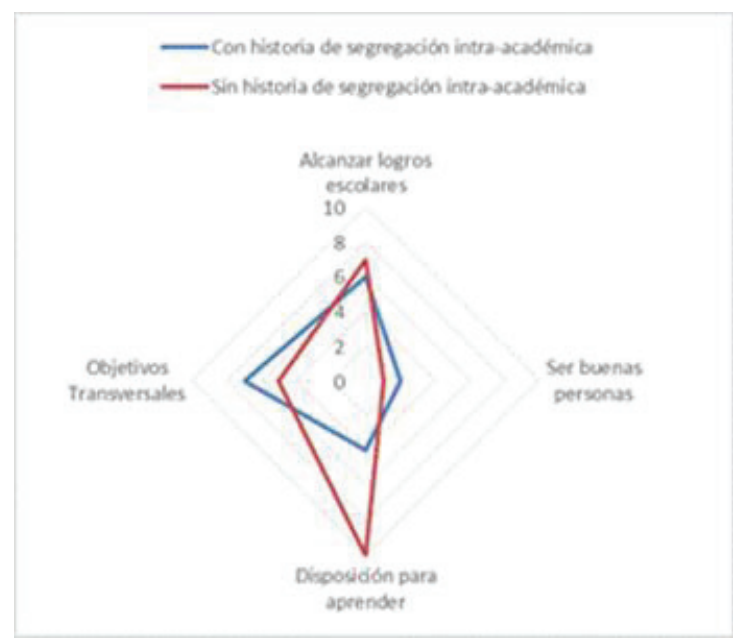

Figura 1. Referencias a Metas de aprendizaje por historia de segregación intra-académica

Se observa que (Figura 1) las referencias de Alcanzar logros escolares y Objetivos Transversales son metas más reiteradas en las Escuelas con historia de Segregación intraacadémica, en tanto las escuelas sin historia de segregación intra-académica reiteran como meta de aprendizaje la promoción de la disposición para aprender. 


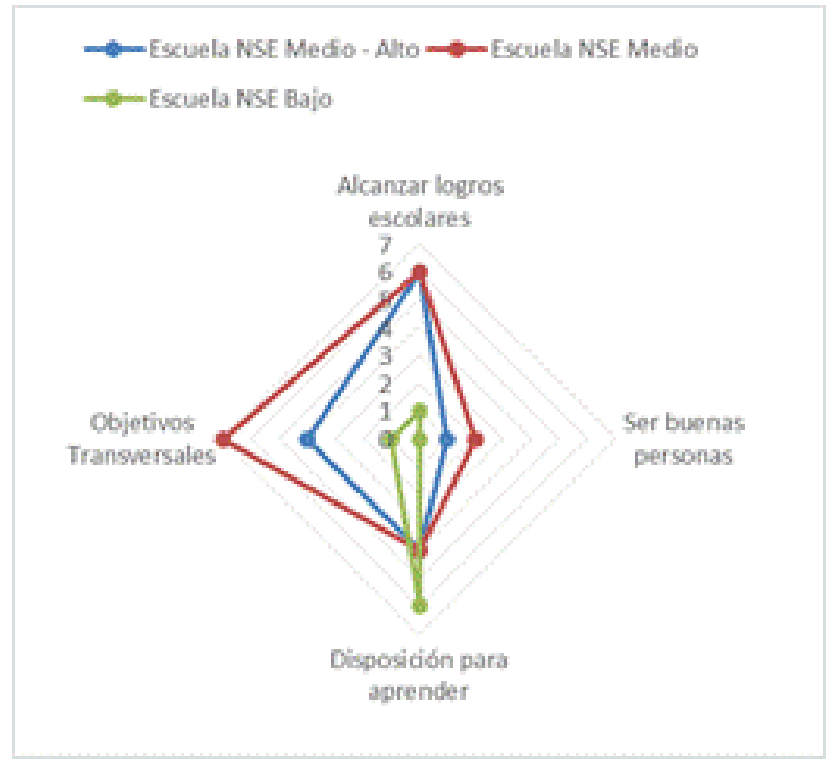

Figura 2. Referencias a Metas de aprendizaje por NSE de las Escuelas

Se observa que (Figura 2) la referencia a la meta de Alcanzar logros escolares tiende a ser mayoritaria en la Escuela de NSE Medio-Alto; en tanto que la referencia a dicha meta y Objetivos Transversales son metas más frecuentes en las Escuelas de NSE Medio; y la Disposición para Aprender es la opción hegemónica en la escuela de NSE Bajo.

\subsubsection{La Diversidad de los alumnos}

La Diversidad de los alumnos, es otro aspecto que incide en el discurso y refiere a tres dimensiones: como posibilidad educativa, como desafío pedagógico y como condición de carencia.

Diversidad como posibilidad educativa, Referencias a la diversidad de los estudiantes como oportunidad de conocer otras realidades o el desarrollo de nuevas formas de abordar el proceso educativo. Por ejemplo:

“...tenemos que convivir adentro de la sala de clases con niños que van de vacaciones al extranjero y otros que no conocen las vacaciones y van mezclándose dentro del aula y van generándose conflictos, pero también se va generando inclusión, que es lo que finalmente buscamos" (Directivo Escuela SPQ, NSE Medio, SIMCE Bajo).

Diversidad como desafío pedagógico, Referencias a la diversidad de los alumnos como un desafío para el cual se buscan o se han intentado diversas acciones de trabajo en el aula adaptadas a las realidades observadas con resultados contingentes en el logro de aprendizajes. Por ejemplo, 
“... yo siento que si a veces ellos son dispersos, yo trato muchas veces de hacer una clase ordenada, pero yo siento que para ellos les resulta fome, no quiero decir con esto que mis clases son un circo y que están llenas de emociones ni nada, pero yo siento que a ellos no les gusta, no son como...no se pueden quedar tranquilos, entonces no puedo hacer una clase en la que ellos sean 100\% autónomos, en las que ellos puedan trabajar siempre solos...en matemáticas estoy yo siempre adelante y las respuestas las hacen solos, pero están "pasemos a la otra, pasemos a la otra" es 50 y 50, guiada y autónoma” (Profesora Novata, Escuela VL, NSE Bajo, SIMCE Bajo)

Diversidad como carencia, Refiere a la diversidad como la ausencia de un atributo de integración social (nivel cognitivo, capital cultural, vulnerabilidad social) que define la frontera de acción de la escuela. Por ejemplo,

“...igual hay niños que necesitan mucha atención, en esta clase hay como 6 que trabajan en el aula de recursos, pero no tenemos ningún caso de Síndrome de Down" (Profesora experta SSF, NSE Medio, SIMCE Alto).

Se organizaron las referencias según la frecuencia en cada categoría en relación con la historia de segregación intra-académica (Figura 3), y el NSE de las escuelas (Figura 4) de modo de poder visualizar las tendencias del discurso.

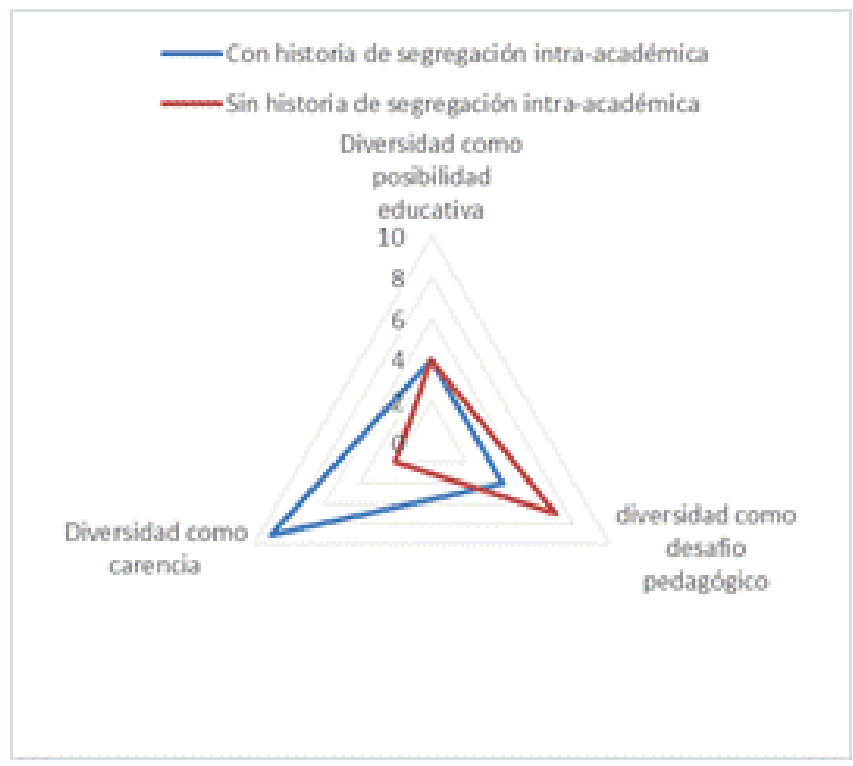

Figura 3. Referencia a la Diversidad de los alumnos según historia de segregación intra-académica 
Se observa que (Figura 3) la diversidad es descrita como carencia de algún tipo en las escuelas con historia de segregación intra-académica, en tanto que la Diversidad es referida como desafío pedagógico de modo mayoritario en el discurso de escuelas sin historia de segregación.

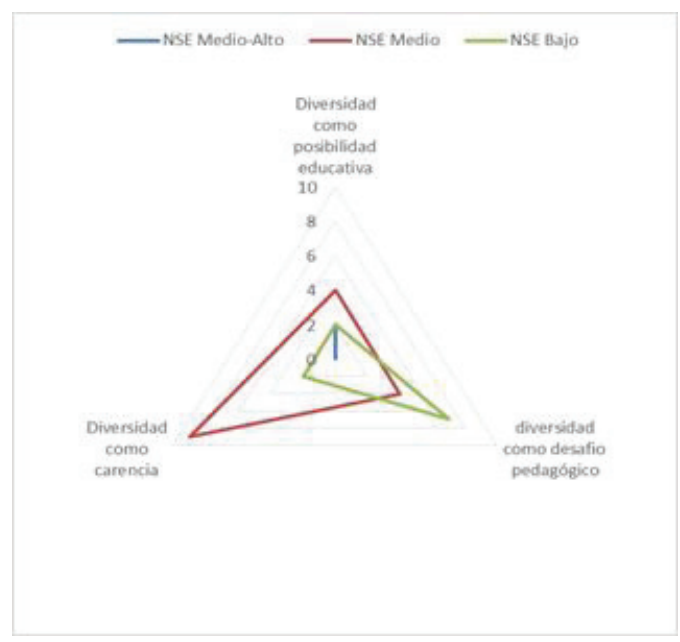

Figura 4. Referencia a la Diversidad de los alumnos según NSE

Se observa que (Figura 4) la referencia a la diversidad como carencia es mayoritaria en el discurso de las escuelas de NSE Medio, en tanto Diversidad como desafío pedagógico es mayoritario en la escuela de NSE Bajo; en tanto, en la escuela de NSE Medio-Alto, la diversidad tiene solamente dos referencias que valoran la diversidad como posibilidad educativa.

\section{DISCUSIÓN DE RESULTADOS}

La práctica pedagógica de aula responde a su contexto inmediato. Esto confirma lo señalado en estudio realizado por De Gregorio y Bruns sobre la variación de la práctica pedagógica incluso en una misma escuela (De Gregorio y Bruns 2016). Se observan más estructuras de intercambio en las profesoras clasificadas como novatas que en las profesoras expertas. Esto confirma investigaciones que encuentran que las profesoras las profesoras novatas presentan un perfil de acción pedagógica que favorece las oportunidades de participación de sus alumnos (Sadik y Sadik 2014) lo que aquí se traduce en estructuras de intercambio. Y esto parece ser así independiente del nivel socioeconómico de la escuela.

No obstante, a pesar de la diferencia de tiempo en los Intercambios en el aula, las profesoras dedican tiempo equivalente para desarrollar criterios EAM Universales, los cuales son diferentes entre las escuelas según su nivel socioeconómico y están asociadas al logro SIMCE. Independiente del NSE las escuelas con Alto SIMCE están fuertemente 
asociadas al EAM Universal Trascendencia, el cual a su vez está asociado al intercambio Colaborativo, en tanto las escuelas de bajo SIMCE están asociadas al EAM Universal Intencionalidad, el cual está asociado al intercambio Expositivo.

No obstante, el logro SIMCE es la identidad pedagógica de la clase social: es más definido en la Escuela de NSE Bajo cuya historia de logro escolar es de bajo logro SIMCE, y en la Escuela Medio-Alto, cuya historia de logro escolar es de Alto SIMCE; pero contingente en el NSE Medio, donde hay una escuela de Alto y otra de Bajo SIMCE. Esto se pone en evidencia en el discurso sobre las Metas de Aprendizaje y la Diversidad de los alumnos; para las escuelas de los extremos socioeconómicos las metas de aprendizaje apuntan con mayor consenso a promover disposiciones para aprender en la escuela de NSE Bajo, y obtener logros escolares en escuela de NSE Medio-Alto; y, en función de dicha meta, ambas escuelas valoran la diversidad como un desafío pedagógico -cada cual ajustado a su realidad-, en tanto que en las escuelas de NSE Medio no hay un claro consenso sobre una meta de aprendizaje específica, y la diversidad es vista como una carencia del entorno, que evidentemente afecta la práctica pedagógica.

Estos resultados grafican modalidades diferentes del concepto que Basil Berstein denominaba dispositivo pedagógico, regulador simbólico de la conciencia, a través de sistema de tres reglas: (1) distributiva, que regula las relaciones entre el poder para distribuir formas de conciencia a través del conocimiento especializado y la forma de acceder al mismo; (2) de recontextualización, que regula la formación del discurso pedagógico donde se integra el discurso del conocimiento especializado a un discurso que crea el orden social específico; y (3) regla de evaluación, que condensa el significado del dispositivo pedagógico para constituir la práctica pedagógica a ser evaluada continuamente (Bernstein 1998). El concepto de dispositivo pedagógico permite comprender que los modos de socialización diferenciados por niveles socioeconómicos disponen a los alumnos de modo desigual a los requisitos para adquisición de conocimientos que tiene la escuela (Rochex 2015), poniéndose de manifiesto en las formas de dialogar y mediar el aprendizaje de la autonomía en el aula y discursos sobre las metas y la diversidad del alumnado en práctica pedagógica.

Desde el concepto de dispositivo pedagógico se puede comprender que en las escuelas Nivel Socioeconómico Bajo, la regla de distribución de la conciencia y poder social de la escuela tome forma de intercambio Expositivo, caracterizado por la comunicación unidireccional; donde la regla de contextualización del quehacer pedagógico de la profesora busca reducir la distancia entre los saberes curriculares y la vida cotidiana de los alumnos a través de explicitar la intencionalidad y promover reciprocidad de la acción pedagógica en el aula para construir y validar el poder estructurante del conocimiento que transmite la escuela. La regla de evaluación, aquella que define la práctica pedagógica, permite comprender que en la Escuela de NSE Bajo se valore como meta de aprendizaje el promover en los alumnos la disposición a adquirir los saberes culturales, donde la diversidad es un dato de la realidad para orientar la acción pedagógica. En síntesis, el dispositivo pedagógico se expresa en una práctica pedagógica efectiva que podemos denominar como de nivelación cultural. Y esta realidad aporta a entender el bajo logro SIMCE.

En tanto el dispositivo pedagógico de la Escuela de nivel socioeconómico MedioAlto, la distribución del conocimiento especializado posiblemente se ve favorecido por la socialización extraescolar de los alumnos, puesto de manifiesto en los intercambios Colaborativos, donde dichos saberes compartidos entre educadora y alumnos se dialogan en una relación más simétrica; asimismo la recontextualización del discurso pedagógico 
posiblemente se ve favorecida la cercanía entre el saber escolar y la socialización de los alumnos, haciendo posible insertar el conocimiento especializado desde la situación del aula hacia los hechos de la vida cotidiana a través de la mediación de la trascendencia, validando recíprocamente ambos mundos, el escolar y el social. La regla de evaluación de la práctica pedagógica en la escuela de NSE Medio-Alto privilegia como meta de aprendizaje el logro escolar, que fortalece y proyecta la propia socialización, donde el discurso sobre la diversidad de los alumnos tiende a ser visto como una posibilidad educativa. En síntesis, para la escuela de NSE Medio-Alto, el dispositivo pedagógico se expresa en una práctica pedagógica efectiva que denominamos como de enriquecimiento cultural. Dicha realidad aporta a entender el alto logro SIMCE.

Respecto a las dos escuelas de NSE Medio, cada una se ubica en los dispositivos pedagógicos de nivelación y de enriquecimiento cultural. Acá la diferencia de logro escolar SIMCE - uno alto y otro de bajo logro-, y similar estrategia para alcanzarlo ambas con historia de segregación intra-académica-, pone en evidencia una situación de diversidad de socialización de los estudiantes, unos con más y otros con menos capital cultural para la integración social, puestas de manifiesto en las aulas, y, en consecuencia de cierta vulnerabilidad de la identidad pedagógica, donde posiblemente co-existen ambos dispositivos pedagógicos en la misma escuela -eso explicaría la segregación intraacadémica-; un discurso ambivalente respecto a las metas de aprendizaje y una percepción de la diversidad de los alumnos como carencia que afecta la acción pedagógica. En este contexto, el dispositivo pedagógico comparte que la práctica pedagógica busque el logro escolar, pero la situación de diversidad del alumnado exige que esta sea evaluada según la realidad de cada grupo de alumnos.

El estudio de Treviño indicaba que la heterogeneidad de los alumnos es un factor que explica la segregación intra-académica (Treviño, Valenzuela y Villalobos 2014a). El presente estudio aporta que, en una perspectiva socio-histórico-cultural, la segregación intra-académica tiene relación con la coexistencia de diferentes dispositivos pedagógicos, que ponen en evidencia la desigualdad social de los estudiantes para responder a las exigencias académicas de la escuela y desafía a la práctica pedagógica de aula la promoción de la autonomía del aprendizaje de los alumnos que a la vez sea inclusiva. En tanto que la cierta homogeneidad de las escuelas de NSE Medio-Alto -cuyos alumnos son similares respecto al bienestar sociocultural-, y de la escuela de NSE Bajo -cuyos alumnos son similares respecto a sus condiciones de vulnerabilidad social-, no requieren de estrategias de segregación intra-académica, y su historia de logros escolares es coherente con las condiciones socioculturales para alcanzarlos.

\section{CONCLUSIONES}

El discurso y práctica pedagógica eficaz está asociada a la historia institucional de logros escolares y al contexto socioeconómico de las escuelas. Específicamente, la práctica pedagógica eficaz para promover el aprendizaje autónomo se sustenta en los recursos culturales que los alumnos traen al aula.

Así, para en la escuela de NSE bajo se reconoce la práctica pedagógica denominada de nivelación cultural, caracterizada por mediación de la Intencionalidad desarrollada preferentemente a través de intercambios expositivos para promover el aprendizaje 
autónomo. En la escuela de NSE Medio-Alto se reconoce la práctica pedagógica efectiva denominada de enriquecimiento cultural, caracterizada por mediación de la Trascendencia desarrollada preferentemente a través de intercambios colaborativos para promover el aprendizaje autónomo. En las escuelas de NSE Medio las prácticas pedagógicas son de nivelación y enriquecimiento cultural.

Entre las limitaciones del estudio se encuentra la ausencia de otros elementos del contexto social que permitan comprender la institucionalidad escolar como marco estructural de lo que sucede en el aula. Además del nivel socioeconómico, aspectos socioculturales como el contexto urbano o rural, o las formas de administración de la escuela -particular o municipal-, es recomendable que sean considerados en futuros estudios.

\section{REFERENCIAS BIBLIOGRÁFICAS}

Agencia de Calidad de la Educación. (2015). Reporte de Calidad. Evolución de los indicadores de calidad de la educación en Chile. Recuperado el 1 diciembre del 2017 en: http://www. agenciaeducacion.cl/wpcontent/uploads/2016/02/Estudio_Reporte_de_calidad.pdf

Assael, C. (2015). La construcción del otro desde los discursos e interacciones de docentes de Eduación Básica, trabajando en aulas regulares con diversidad cognitiva y diversidad étnica. Búsqueda de conexiones entre la denominada educación Inclusiva y la educación intercultural. Tesis de Doctorado en Ciencias de la Educación, Universidad de Santiago de Chile, Santiago, Chile.

Bernstein, B. (1998). Pedagogía, control simbólico e identidad: teoría, investigación y crítica: Morata.

Bruns, B, Luque, J., De Gregorio, S., Evans, D., Fernández, M., Moreno, M., Rodríguez, J., Toral, G. y Yarrow, N. (2014). Profesores excelentes: cómo mejorar el aprendizaje en América Latina y el Caribe: Banco Mundial.

Cornejo, R. y Redondo, J. (2007). Variables y factores asociados al aprendizaje escolar. Una discusiòn desde la investigación actual. Estudios Pedagógicos, 32(2), 155-175.

Cuadrado, I. y Fernández, I. (2008). ¿Cómo intervienen maestros y profesores para favorecer el aprendizaje en Secundaria? Un estudio comparativo desde el análisis del discurso. Infancia y Aprendizaje, 31(1), 3-23.

Damianova, M. y Sullivan, G. B. (2011). Rereading Vygotsky's theses on types of internalization and verbal mediation. Review of General Psychology, 15(4), 344-350. doi:10.1037/a0025627

De Gregorio, S. y Bruns, B. (2016). Práctica docente en el aula: una mirada al interior de las salas de clases en América Latina y el Caribe. In J. Manzi y M. R. García (Eds.), Abriendo las puertas del aula. Transformación de las prácticas docentes (pp. 59-92). Santiago, Chile: Ediciones Universidad Católica de Chile.

Donoso, S. y Arias, Ó. (2012). Distribución desigual de las oportunidades educativas en el territorio y migración de la matrícula escolar: el caso de la región de Los Lagos (Chile). Estudios pedagógicos, 38(2), 35-54.

Emanuelsson, J. y Sahlström, F. (2008). The Price of Participation: Teacher control versus student participation in classroom interaction. Scandinavian Journal of Educational Research, 52(2), 205-223. doi:10.1080/00313830801915853

Feuerstein, R. (1990). The theory of structural cognitive modifiability. In B. Z. Presseisen, R. J. Sternberg, K. W. Fischer, C. C. Knight, y R. Feuerstein (Eds.), Learning and Thinking Styles: Classroom Interaction (pp. 68-134). Washington, DC: National Education Association.

Feuerstein, R. (2006). Instrumental Enrichment. Jesuralem, Israel: ICEL, Publications.

Feuerstein, R., Rand, Y., Hoffman, M. B., Egozi, M. y Shachar-Segev, N. (1991). Instrumental 
Enrichment Program. In L. Idol y B. Jones (Eds.), Educational Values and Cognitive Instruction (Vol. 2, pp. 139-178). Hillsdale, N.J: Erlbaum.

Godoy, F., Varas, L., Martínez, M., Treviño, E. y Meyer, A. (2016). Interacciones pedagógicas y percepción de los estudiantes en escuelas chilenas que mejoran: una aproximación exploratoria. Estudios Pedagógicos,. 42(3), 149-169.

Hennessy, S., Rojas-Drummond, S., Higham, R., Márquez, A., Maine, F., Ríos, R. García-Carrión, R., Torreblanca, O. y Barrera, M. (2016). Developing a coding scheme for analysing classroom dialogue across educational contexts. Learning, Culture and Social Interaction, 9, 16-44. doi:10.1016/j.lcsi.2015.12.001

Kozulin, A. (1999). Sociocultural Contexts of Cognitive Theory. Human Development (0018716X),42(2), 78-82. doi:10.1159/000022612

Laboratorio Latinoamericano de Evaluación de la calidad de la Educación. (2010). Factores asociados al logro cognitivo de los estudiantes de América Latina y el Caribe. Santiago, Chile: Oficina Regional de Educación de la UNESCO para América Latina y el Caribe (OREALC/UNESCO Santiago) y del Laboratorio Latinoamericano de Evaluación de la Calidad de la Educación - LLECE.

Landis, J. y Koch, G. (1977). The measurement of observer agreement for categorical data. Biometrics, 33(1), 159-174.

Lehesvuori, S. (2013). Towards Dialogic Teaching in Science. Challenging Classroom Realities through Teacher Education. (Doctoral thesis), University of Jyväskylä, Jyväskylä, Finland. (465)

Martinic, S. y Villalta, M. (2016). Jornada Escolar Completa y organización del tiempo en la sala de clases de Educación Básica. In J. Manzi y M. R. García (Eds.), Abriendo las puertas del aula. Transformación de las prácticas docentes (pp. 317-348). Santiago, Chile: Ediciones Universidad Católica de Chile.

Moreno, R. y Martínez, R. (2007). Aprendizaje autónomo. Desarrollo de una definición. Acta Comportamentalia, 15(1), 51-62.

Murillo, F. y Román, M. (2009). Mejorar el desempeño de los estudiantes de América Latina. Revista Mexicana de Investigación Educativa, 14(41), 451-484.

Nathan, M. y Kim, S. (2009). Regulation of Teacher Elicitations in the Mathematics Classroom. Cognition and Instruction, 27(2), 91-120. doi:10.1080/07370000902797304

Navarro, P. y Díaz, C. (1999). Análisis de Contenido. In J. M. Delgado y J. Gutierrez (Eds.), Métodos y técnicas cualitativas de investigación en ciencias sociales (3 ed., pp. 177-224). Madrid: Alianza Editorial.

Orrú, S. (2003). Reuven Feuerstein y la Teoría de la Modificabilidad Cognitiva Estructural. Revista de Educación, 33(2), 33-54.

Osses, S. y Jaramillo, S. (2008). Metacognición: un camino para aprender a aprender. Estudios Pedagógicos,34(1), 187-197.

Piñuel, J. (2002). Epistemología, metodología y técnicas del análisis de contenido. Sociolinguistic Studies, 3(1), 1-42.

Poehner, M. y Infante, P. (2015). Mediated Development: Inter-psychological activity for L2 education. Language and Sociocultural Theory, 2(2), 161-183. doi:10.1558/lst.v2i2.26982

Rimmele, R. (2009). What is Videograph? (Version 4.2.1.25X3 from September 2013) [Videograph]. Kiel, Alemania: IPN Leibniz-Institut für die Pädagogik der Naturwissenschaften an der Universität Kiel. Retrieved from http://archiv.ipn.uni-kiel.de/projekte/videograph/enhtmStart.htm

Rochex, J.-Y. (2015). Desigualdades escolares, herramientas para evaluarlas y estudios de los procesos de producción. Profesorado. Revista de currículum y formación del profesorado, 19(3), 7-25.

Sadik, S. y Sadik, F. (2014). Investigating Classroom Management Profiles of Pre-service Teachers (Cukurova University Sample). Procedia - Social and Behavioral Sciences, 116, 2369-2374. doi: 10.1016/j.sbspro.2014.01.575

Sánchez, G. y Amigo, X. (2016). El contexto y los actores de la práctica: estudio sobre la representación del profesor en formación. Páginas de Educación, 9(2), 118-155. 
Shreyar, S., Zolkower, B. y Perez, S. (2010). Thinking aloud together: A teacher's semiotic mediation of a whole-class conversation about percents. Educational Studies in Mathematics, 73(1), 21-53. doi:10.1007/s10649-009-9203-3

Tébar, L. (2007). El profesor mediador del aprendizaje (1 ed.). Santiago de Chile: Arrayan Editores.

Tocaimaza-Hatch, C. (2016). Mediated Vocabulary in Native Speaker-Learner Interactions During an Oral Portfolio Activity. Foreign Language Annals, 49(2), 336-354. doi:10.1111/flan.12190

Tornero, B., Ramaciotti, A., Truffello, A. y Valenzuela, F. (2015). Nivel cognitivo de las preguntas que formulan las educadoras de párvulos. Educación y Educadores, 18(2), 261-283. doi:10.5294/ edu.2015.18.2.5

Treviño, E., Valenzuela, J. y Villalobos, C. (2014a, mayo, 2014). ¿Se agrupa o segrega al interior de los establecimientos escolares chilenos? Segregación académica y socioeconómica al interior de la escuela. Análisis de su magnitud, principales factores explicativos y efectos. Nota técnica FONIDE N $N^{o}$ : F711296, CPCE y CIAE., 26, 12.

Treviño, E., Valenzuela, J. y Villalobos, C. (2014b). Segregación académica y socioeconómica al interior de la escuela. Análisis de su magnitud, evolución y principales factores explicativo. Santiago: Fondo de Investigación y Desarrollo en Educación - FONIDE.

Treviño, E., Varas, L., Godoy, F., y Martinez, M. (2016). ¿Qué caracteriza a las interacciones pedagógicas de las escuelas efectivas chilenas?: una aproximación exploratoria. In J. Manzi y M.

R. García (Eds.), Abriendo las puertas (Vol. 1, pp. 185-220). Santiago: Ediciones Universidad Católica de Chile.

Tzuriel, D. (2010). Programa de Intervención basado en la propuesta de aprendizaje mediado. Paper presented at the Familias y Jardín Infantil: Juntos aseguran el futuro, Santiago, Chile. http:// www.unicef.cl/web/wp-content/uploads/doc_wp/WD\%2012-Hogar\%20de\%20Cristo.pdf

Tzuriel, D. y Shamir, A. (2007). The effects of Peer Mediation with Young Children (PMYC) on children's cognitive modifiability. British Journal of Educational Psychology, 77, 143-165. doi:10.1348/000709905x84279

UNICEF. (2004). ¿Quien dijo que no se puede? Escuelas efectivas en sectores de pobreza. Santiago: UNICEF.

Vidal, D. (2009). No interior da sala de aula: ensaio sobre cultura e prática escolares. Curriculo sem Fronteiras, 9(1), 25-41.

Villalta-Paucar, M. A., Martinic-Valencia, S., Assael-Budnik, C. y Aldunate Ruff, N. (2018). Presentación de un modelo de análisis de la conversación y experiencias de aprendizaje mediado en la interacción de sala de clase. Revista Educación, 42(1), 87-104. doi:10.15517/revedu. v42i1.23431

Villalta, M. y Palacios, D. (2014). Discurso y práctica pedagógica en contextos de alto rendimiento escolar. Estudios Pedagógicos, 40(2), 373-389.

Villalta, M. (2009). Análisis de la conversación. Una propuesta para el estudio de la interacción didáctica en sala de clase. Estudios Pedagógicos, 35(1), 221-238

Villalta, M. (2014). Aprendizaje Escolar y demanda cognitiva en la sala se clase T. Mourinho, F. Miranda, M. Berdullas, C. Biscaia, S. Saunders, D. Dias, T. Lopes, T. Espassandim, C. Pita, T. Pereira, y C. Janeiro (Eds.), Actas do IX Congresso Iberoamericano de Psicologia/2 ${ }^{\circ}$ Congresso da Ordem dos Psicólogos Portugueses (pp. 1226-1238). Retrieved from https://www. ordemdospsicologos.pt/ficheiros/documentos/livro_de_actas_2congressoopp.pdf.

Villalta, M. (2017). Interacción profesor-alumnos y promoción del aprendizaje en escuelas de Educación Básica con diferente desempeño escolar. Revista de Pedagogía, 38(102), 80-96.

Villalta, M. y Martinic, S. (2013). Interacción Didáctica y procesos cognitivos. Una aproximación desde la práctica y discurso docente. Universitas Psychologica, 12(1), 221-233. Retrieved from 1657-9267(201303)12:1<221:IDYPCA>2.0.TX;2-X).

Villalta, M., Martinic, S. y Assael, C. (2013). Conocimiento escolar y procesos cognitivos en la interacción didáctica de sala de clase. Perfiles educativos, 35(141), 84-96. 
Volante, P., Cumsille, P., Denardin, F. y Müller, M. (2008). Análisis del cambio en los niveles de logro de Escuelas de alta vulnerabilidad social. Estudios Pedagógicos, 34(2), 179-191.

Wells, G. y Mejia, R. (2005). Toward dialogue in the classroom: learning and teaching through inquiry Working Papers on Culture, Education and Human Development, 1,(4) 1- 45. Universidad Autónoma de Madrid. 